\title{
El método científico y su aplicación en las Ciencias de la Información (Relaciones Públicas)
}

\author{
Luis Felipe SOLANO \\ Universidad Complutense de Madrid \\ lfsolano@yahoo.es
}

Recibido: $16 / 02 / 2011$

Aceptado: 21/02/2011

\section{RESUMEN}

Tras un somero análisis sobre el método científico y su aplicación tanto a las Ciencias de la Naturaleza como a las Ciencias de la Cultura, el presente artículo de investigación pretende establecer qué método o, mejor dicho, qué métodos son los más adecuados para las ciencias que nos ocupan, es decir, las Ciencias de la Información, en tanto en cuanto se trata de Ciencias Sociales, Culturales, valorativas y finalistas, y más concretamente, las Relaciones Públicas como conjunto de saberes nacidos de la práctica y para la práctica como muchos afirmamos. Así mismo, se abordan cuáles serían los fundamentos y principios de los que, a nuestro juicio, deberían partir las Relaciones Públicas a la hora de investigarlas desde la reflexión teorética. Palabras clave: Método científico, Ciencia Natural, Ciencia Cultural, Relaciones Públicas.

The scientific method and its application in information science (Public Relations)

\begin{abstract}
After a exhaustive analysis of the scientific method and its application to the natural sciences as the science of culture, this article tries to establish what methods are best suited for the sciences that concern us, i.e. information science, as in both, it is social science, culture, values and purposes, more specifically, public relations as a set of knowledge born of practice and practice as many assert, likewise, will be approached which are the bases and principles that we believe. public relations should be based when investigated from theoretical reflection.
\end{abstract}

Key words: Scientific method, Natural Science, Cultural Science, Public relations.

\section{INTRODUCCIÓN}

Sí, ineludible, siquiera se trate, como ya han denunciado multitud de estudiosos del tema, de una ilusión más que de una realidad ${ }^{1}$. No obstante, una tradición inveterada en todo trabajo de investigación nos obliga a afrontar su estudio, siquiera perfilando su auténtica naturaleza y alcance, cuestión que en el campo de las ciencias sociales alcanza

\footnotetext{
${ }^{1}$ Entre ellos, Mario Bunge.
} 
superlativa importancia, dado que en muchos supuestos el método y la teoría sustantiva se funden y confunden, resolviéndose en un solo "corpus" de conocimientos ${ }^{2}$.

Ahora bien, en qué consiste el método científico, o dicho de otro modo, qué se entiende por método científico.

Ferrater Mora, en su "Diccionario de Filosofía", dice textualmente:

Se tiene un método cuando se sigue un cierto "camino", "jodós", para alcanzar un cierto fin, propuesto de antemano como o "vital"; por ejemplo, la "felicidad". En ambos casos hay, o puede haber, un método.

Y más adelante, continúa nuestro autor,

El método se contrapone a la suerte y al azar, pues el método es ante todo un orden manifestado en un conjunto de reglas. Se podría alegar que si la suerte y el azar conducen al mismo fin propuesto, el método no es necesario, pero se ha hecho observar que: (1) tal. Este fin puede ser el conocimiento o puede ser también un "fin humano" ni la suerte ni el zar suelen conducir al fin propuesto; (2) un método adecuado no es sólo un camino, sino un camino que suele abrir otros, de tal modo que o se alcanza el fin propuesto más plenamente que por medio del azar y la suerte, o se alcanzan inclusive otros fines que no se habían precisado... (3) el método tiene, o puede tener, valor por sí mismo ${ }^{3}$.

Sin embargo, no nos queda más remedio que advertir que una amplia corriente de filósofos rechaza abiertamente incluso la existencia de un método científico ${ }^{4}$; de ahí que, en la actualidad, se prefiera hablar de "estrategias de investigación" antes que de método, reduciendo el contenido material de éste a un conjunto de reglas que hacen posible alcanzar un cierto grado de probabilidad en los descubrimientos que se efectúan en el campo de las ciencias.

Sea como fuere, es lo cierto que, en última instancia, el método científico ha gozado y goza de un prestigio singular, avalado sin duda por el hecho de que se trata de la plasmación de un sueño: disponer de un sistema que garantiza casi automáticamente el hallazgo de nuevas leyes científicas ${ }^{5}$.

${ }^{2}$ En general, todas las corrientes sociológicas y ello es así por una razón muy sencilla: al carecer la sociología de objeto de estudio - cada escuela lo configura a su arbitrio- y al ser tributario el método de investigación del objeto a estudiar, los métodos varían extraordinariamente de unas tendencias a otras, lo que a la postre determina que método y teoría se resuelvan en un corpus de conocimientos entremezclados y en los que resulta imposible separar lo que corresponde a la teoría sustantiva de lo que pertenece a 1 ámbito metodológico.

${ }^{3}$ Véase en op. cit., voz "método".

${ }^{4}$ Entre ellos y como figura destacada dentro del ámbito neopositivista lógico, Mario Bunge, quien no vacila en rechazar de plano la existencia de una especie de "varita mágica" que, aplicada al estudio de un objeto, genera casi automáticamente leyes científicas.

${ }^{5}$ Recordemos que el fin de la ciencia es la formulación de leyes científicas, esto es, relaciones de invariancia interfenoménica, o más sencillamente, lo permanente en el mudar. 
Resulta curioso comprobar cómo a lo largo de la historia de la ciencia los autores que han hablado y escrito sobre el método científico han sido en su inmensa mayoría teóricos, es decir, personas que nunca lo han puesto en práctica; sin embargo, los que de verdad lo han aplicado, fundamentalmente desde Galileo hasta hoy, nunca han escrito una sola línea acerca de él.

No obstante lo dicho más arriba, el respeto a la tradición nos impone dedicar algunas páginas al examen de lo que los estudiosos de estas cuestiones han dicho acerca del método científico.

En primer lugar, observemos que las cuestiones relativas al método son muy antiguas, siquiera la investigación respecto de su naturaleza y formas y el auge que la misma adquiere corresponden a la edad moderna, cuando, como quiere Ferrater Mora, "se pretende encontrar un 'método de invención' distinto de la mera 'exposición' y de la simple 'prueba de lo ya sabido'",

No resistimos a la tentación de transcribir la definición que del método científico nos ofrece Descartes:

El arte de bien disponer una serie de diversos pensamientos, ya sea para descubrir una verdad que ignoramos, ya para probar a otros una verdad que conocemos ${ }^{7}$.

Aunque para Descartes el método no sólo es un procedimiento para descubrir -"ars inveniendi"-, sino también para demostrar -"ars demostrandi"-, e incluso para exponer "ars exponiendi"-, no tenemos más remedio que reconocer que la mayoría de los autores consideran exclusivamente o casi exclusivamente la primera parte, dejando para un lugar muy secundario el "ars exponiendi" o "demostrandi".

Cuatro reglas debe cumplir en el pensamiento del filósofo francés el método científico y pese a los años transcurridos desde que las formulara continúan gozando de plena vigencia. Dichas reglas son:

1) No tener jamás una cosa por verdadera, sin que conste evidentemente que lo es; es decir, evitar con todo cuidado la precipitación y la prevención, y no incluir nada más en los juicios que lo que se presente tan clara y distintamente al espíritu, que no ocurra ninguna razón u ocasión de ponerlo en duda. 2) Dividir cada una de las dificultades que se examinen en tantas partes como se pueda, y cuantas sean menester para mejor resolverlas. 3) Llevar orden en los propios pensamientos comenzando por los objetos más simples y fáciles de conocer, para subir poco a poco y como por grados, hasta el conocimiento de los más complejos. 4) Hacer siempre divisiones tan enteras y revistas tan generales, que quede uno seguro de no omitir nada. ${ }^{8}$

Quedaría harto incompleta esta sumaria descripción de la evolución histórica de las reflexiones sobre el método científico si no recordáramos junto a Descartes y Pascal a

\footnotetext{
${ }^{6}$ Cfr. "Diccionario de Filosofía", voz "método".

${ }^{7}$ Cfr. "Logique de Port Royal", Parte IV, Cap. 2. Se trata de un calco de la obra de Descartes.

${ }^{8}$ Cfr. "Le Discours de la Methode".
} 
figuras tan importantes como Leibniz, Bacon y entre nosotros, incluso bastantes años antes, al polifacético Ramón Llul, quien dedicó algunas de sus más de ciento veinticinco obras al estudio del método.

\section{El método científico: deductivo e inductivo}

Cuando se habla de los procedimientos que utiliza la ciencia para alcanzar sus objetivos, se suelen unificar estos bajo la denominación genérica de método científico, ofreciendo al profano la falsa imagen de una singularidad que dista mucho de ser verdad. No existe un método científico sino métodos científicos, tan diversos como lo son el tipo de objeto que estudian y la peculiaridad o propósito del estudio en cuanto tal. Ya inicialmente nos encontramos con una división de gran importancia: la que distingue entre método deductivo y método inductivo; el primero parte de los grandes principios generales para aplicarse a los casos concretos y es el propio de las ciencias que constituyen intelectualmente su objeto como la matemática y el Derecho; el segundo, parte del estudio de los objetos singulares, de los que induce a la postre un principio general, y es el propio de las ciencias naturales. Método deductivo y método inductivo son, pues, el método científico por antonomasia, siquiera debamos registrar el dato de que los grandes teóricos de la metodología sólo admitieron como único y verdadero método científico -Descartes, Leibniz, etc.- el método deductivo, ya que es el único que proporciona un conocimiento cierto de la realidad que estudia.

El pueblo griego destacó en la aplicación del método deductivo y de ahí sus grandes éxitos en la matemática, en la geometría, etc., es decir, en aquellas ciencias formales que al constituir intelectivamente su objeto hacía posible la aplicación de la deducción partiendo de los axiomas y los postulados. El fracaso de la ciencia griega tuvo lugar cuando se intentó aplicar el método deductivo al estudio de la naturaleza, ya que éste exigía la inducción. ${ }^{10}$

Otras clasificaciones metodológicas cabe formular en el tema que nos ocupa, siquiera carezcan de la importancia realmente superlativa de la que acabamos de mencionar; no obstante, consideramos de interés la que distingue entre el método analítico y el método sintético; el primero procede descomponiendo el objeto de estudio en sus diversas partes; el segundo, llevando a cabo un estudio de su totalidad.

En lo tocante al método científico, hay que formular una advertencia importante y es la que deriva de la utilización de un mismo término para designar realidades diversas; nos referimos a la distinción entre el método, los métodos y las llamadas técnicas metodológicas o instrumentales, origen de multitud de confusiones sobre todo para el profano.

${ }^{9}$ Entre ellas, el "Ars Universalis seu Lectura super artem compendiosam inveniendi veritatem”, la "Applicatio Artis Generalis ad varias scientias" y "De experientia realitatis artis generalis". La importancia del genio mallorquín se pone de relieve si pensamos que antecede más de tres siglos a algunos de los autores mencionados.

${ }^{10}$ Asimov, en su Historia de la Ciencia, pone como ejemplo la medición de la distancia entre Atenas y Corinto; esta distancia resultaba imposible de calcular deductivamente, ya que era necesario contar uno tras otro el número de pasos que separaba ambas ciudades. 
Método científico en cuanto tal, como dijimos antes, no hay más que dos, el deductivo y el inductivo, los demás -el método analítico, el sintético, el experimental, etc.- son variaciones sobre un mismo tema, es decir, o se corresponden con el método deductivo o con el inductivo, generalmente con este último. Ahora bien, la incorporación de un determinado método a una ciencia ${ }^{11}$ puede llegar a alcanzar un grado de perfección tan alto que prácticamente pueda hablarse de un método propio; así, por ejemplo, sucede con las Física; hoy puede hablarse con entera propiedad de un método físico de investigación y otro tanto puede decirse de la Bioquímica.

Cuestión distinta a la que acabamos de examinar es la referente a las técnicas metodológicas o instrumentales, las cuales aun pertenecientes como no podía ser menos a un método determinado vienen impuestas por la naturaleza del objeto a estudiar; así, por ejemplo, para el examen de los planetas, es preciso auxiliarse de un telescopio; en cambio, para el estudio de la vida microscópica, es necesario utilizar un microscopio. De modo idéntico sucede con las técnicas de tinción en biología, etc.

Si bien, como acabamos de ver, la naturaleza del objeto condiciona el método a utilizar, no es éste el aspecto más importante a tener en cuenta.

Si bien la ciencia, el conocimiento científico, puede definirse como la búsqueda de la verdad, se trata de una definición tan amplia que prácticamente carece de interés y por lo tanto, exige una serie de matizaciones que la conviertan en operativa. La ciencia es la búsqueda de la verdad, pero de un modo específico, especificidad que comienza con la determinación rigurosa de lo que se entiende por verdad.

Algunos autores han sostenido que la calificación de científico de un tipo de saber conviene sólo a aquel conocimiento que se obtiene por medio del método científico; otros, atribuyen dicha característica a un conocimiento riguroso, objetivo, etc. Sin embargo, a nada que reflexionemos un punto sobre la cuestión, caeremos en la cuenta de que estas adjetivaciones de la ciencia convienen a todo conocimiento, incluso a los que no exigen la utilización del llamado método científico, habida cuenta de que éste, su rigor, su inicial presunción de fiabilidad, etc. no es más que un estadio de su evolución histórica, sujeto siempre a inexorable mutación ${ }^{12}$.

Aunque brevemente apuntado en los párrafos anteriores, no podemos olvidar que el objeto de estudio condiciona el método a emplear, sin embargo no sólo es el objeto en cuanto tal, sino también el propósito o fin del propio investigador el que determina la utilización de un tipo de método y con ello entramos en el problema de la unidad o pluralidad del conocimiento científico. ¿Es la ciencia una o cabe hablar de ciencias, en plural? En puridad de conceptos la ciencia es una en tanto en cuanto con su quehacer lo que se pretende es hallar la verdad; sin embargo, este concepto de lo que es la ciencia es muy superficial; su generalidad lo hace escasa o nulamente operativo; en consecuencia, no nos vale.

\footnotetext{
${ }^{11}$ Más adelante, al ubicar las Relaciones Públicas en el ámbito epistemológico que consideramos le corresponde, volveremos sobre este punto.

${ }^{12}$ Cfr. Bernal, Historia Social de la Ciencia, en la que sostiene como el propio concepto de ciencia se halla sujeto al cambio. Al fin y al cabo, no podemos olvidar que el tiempo es el gran destructor de todo.
} 


\section{Ciencia Natural y Ciencia Cultural}

La ciencia, el conocimiento científico, persigue hallar lo que algunos filósofos de la ciencia han denominado "relaciones de invariancia interfenoménica"13 o de modo más doméstico, más coloquial, "lo permanente en el mudar", es decir, aquello que no cambia, aquello que permanece por encima de los avatares del tiempo y del espacio. Eso y no otra cosa es la ciencia, siquiera se trate de un modo específico de conocer, como hemos tenido ocasión de ver en páginas anteriores. Pero, sucede que ya inicialmente hay dos modos o formas de aproximarse a la realidad; uno, a la realidad en cuanto tal y otro, a la realidad en cuanto significación.

Ambos modos configuran la distinción entre lo que ha dado en llamarse ciencias de la naturaleza y ciencias de la cultura y cuyo antecedente hay que buscarlo en la obra del pensador alemán Wilhelm Dilthey y en concreto en su ya clásica "Introducción a las Ciencias del Espíritu" ", en la que formula la distinción entre ciencias de la naturaleza y ciencias del espíritu. Sin embargo, la influencia que esta distinción iba a ejercer en el pensamiento científico de las generaciones venideras no se iba a deber a su autor, sino más bien a otro filósofo también alemán, pero de muchísimo menor calado intelectual, el profesor Rickert, neokantiano y que tuvo la inmensa fortuna de soslayar ese formidable movimiento filosófico que se llamó Positivismo, movimiento que como han reconocido numerosos autores tuvo la "virtud" de arruinar, al menos en sus inicios, bibliotecas enteras de ciencias sociales. ¿Cuál había sido el acierto de Rickert? Pues muy sencillo, si bien pone de relieve una vez más el poder de la semántica, sustituir la palabra espíritu por la de cultura. El positivista negaba absolutamente la existencia del espíritu y consecuentemente demonizaba todo lo que había tenido o tenía la menor relación con él. Era un término metafísico y la metafísica formaba parte de un estadio ya superado por la humanidad. No obstante, el positivista se encontraba en su camino con factores o ingredientes de muy difícil encaje y que reclamaban una explicación, explicación que el positivista no podía dar. Y he aquí la solución perfecta; el término cultura, una expresión cómoda en la que cabía todo aquello que no se correspondía con los hechos positivos y que carecía de ese tufillo metafísico que tanto molestaba al positivismo.

La distinción de Rickert conoció un éxito sin precedentes, pese a ser, como construcción intelectual, muy limitada y desde luego, carente del vigor y de la originalidad de la de Dilthey.

¿Qué persiguen las ciencias de la naturaleza y en qué se diferencian de las ciencias de la cultura?

Antes dejamos apuntado un dato que se nos antoja de la mayor importancia, siquiera ha pasado desapercibido para algún lector poco avisado. Dijimos, en efecto, que las ciencias naturales constituían como objeto de estudio la realidad en cuanto tal, es decir, como se ofrece a los ojos del observador, y el investigador procede a su descripción e indaga causas y efectos y punto. Las ciencias culturales describen asimismo su objeto

\footnotetext{
${ }^{13}$ Véase Wartofsky, Filosofía de la Ciencia, Alianza Editorial, Madrid.

${ }^{14}$ En alemán, Einleitung im die Geisteswissenshaften.
} 
de estudio, pero trascienden la pura realidad física para entrar en el campo de la significación, en la específica singularidad que encapsula. Así, por ejemplo, y citamos un supuesto clásico, el Moisés de Miguel Angel. Se trata, sin duda, de un bloque de mármol y caben de él dos tipos de descripción; una, en su condición estrictamente física; la otra, en su calidad de obra de arte. Ambas son ciertas, pero no cabe confundirlas. Una correspondería a la ciencia natural; la otra, a la ciencia cultural. Una busca generalizaciones; la otra, singularidades.

Cabe hallar todavía otro factor de distinción entre ambas clases de ciencias. Como afirmamos antes, el científico natural se limita a describir y a determinar causas y efectos. Ahí concluye su labor. El científico cultural da un paso más: formula juicios de valor; su tarea no termina con la actuación equivalente a la de su colega natural, sino que exige un pronunciamiento acerca de la adecuación o inadecuación de su objeto de estudio al valor que pretende incorporar Así, por ejemplo, estudia la organización de la justicia y no se limita a describir los tribunales, las leyes que regulan su funcionamiento, etc., sino que al final se plantea la cuestión de si dichos tribunales son los más adecuados para cumplir el fin al que deben servir, esto es, la más exacta realización de la equidad, o sea, la justicia en el caso concreto.

¿A qué obedece semejante modo de actuar? Pues sencillamente a que los objetos culturales existen en función de su capacidad de servicio al ser humano; son objetos, "cosas" artificiales, cuya razón de ser obedece a que hacen más fácil la vida humana.

Las ciencias culturales son, por consiguiente, aquellas que pretenden conocer el modo más adecuado para la incorporación de un valor, bien sea a un objeto, bien sea a una relación; así, por ejemplo, la jurisprudencia o ciencia del Derecho investiga el modo más adecuado de incorporar el valor justicia a las relaciones humanas; la estética, el de incorporar el valor belleza a las obras o acciones realizadas por el ser humano.

\section{Ciencias de la Información (Relaciones Públicas)}

Por lo que se refiere a las Ciencias de la Información y, en particular, a las Relaciones Públicas, en cuanto conjunto de saberes, éstas estudian o pretenden, como hemos defendido, verificado y publicado en multitud de ocasiones, incorporar a las relaciones humanas un valor, la solidaridad, a través de los correspondientes procesos de comunicación grupal e intergrupal, procesos de comunicación que obviamente tienen mero valor instrumental y se hallan sujetos al albur de la técnica; de ahí, el error en que incurren, si es que cabe hablar de error ( $i !)$, los que pretenden tecnificar las Relaciones Públicas.

Adviértase que esta concepción valorativa y finalista de las Relaciones Públicas ha sido también examinada con detenimiento.

Las Relaciones Públicas, como han subrayado multitud de autores, constituyen un saber nacido de la práctica y para la práctica y en última instancia, apurando los conceptos hasta el límite, serían en el mejor de los casos sociología aplicada.

Recordemos que, de acuerdo con Cassirer, existen dos modos de concebir la realidad: el basado en la causa y el basado en la forma; en el pasado, la filosofía y la ciencia se inclinaron por la forma; la ciencia moderna, se ha inclinado por la causa; sin 
embargo, hoy asistimos a una revisión de ambas posturas, a la que no es ajena la crisis por la que atraviesa la ciencia natural. Si a ello añadimos que las ciencias culturales y la nuestra es un paradigma de esta clase de ciencias, son aquellas en las cuales el empleo del método de la forma permite alcanzar más efectivos resultados, no tiene nada de particular que semejante método haya alcanzado un predominio casi total en la investigación.

De otro lado, Cassirer, autor al que seguimos, afirma que:

....el origen de la función simbólica -que es para él el objeto capital de las ciencias culturales, de acuerdo con su idea del hombre como animal syimbolicum- no es comprensible por medios cientificos, es decir, predominantemente causales. En lo cultural todo devenir permanece dentro de un determinado ser, es decir, dentro de la "forma" en general. Por eso puede ser designado tal devenir, para emplear una expresión platónica, como un "génesis eis oúsian", como un devenir hacia el ser. ${ }^{15}$

Si las Ciencias de la Información forman parte de ese conjunto de saberes culturales, es decir, de las Ciencias de la Cultura, y no de las Ciencias de la Naturaleza, el único método aplicable es indudablemente el deductivo. Ahora bien, si la proposición a dilucidar es de reflexión teorética, el método que debería utilizarse es el denominado lógico-formal, puesto que una vez aceptado el concepto determinado propuesto se alcanzarían una serie de conclusiones válidas siempre y cuando nos moviésemos dentro del marco teórico-conceptual enunciado a priori. Ello no significa que se abandone el campo de la realidad, limitándonos a trazar esquemas ideales absolutamente carentes de utilidad y válidos sólo como "divertimentos" intelectuales, al fin y al cabo, las Relaciones Públicas, como construcción científica, son una disciplina práctica, una ciencia aplicada e ignorar la realidad sería traicionar su objetivo y su razón de ser. Sin embargo, ello tampoco significa que en nuestra concepción del fenómeno olvidemos que, en gran medida, es un saber que, hoy por hoy, construye, como la matemática, el Derecho o la filosofía, intelectivamente su objeto, es decir, inicialmente carece de él y sólo la reflexión del estudioso es la que, creándolo, dota de sentido y construye el lenguaje -toda ciencia es primero de todo un lenguaje- en el que formulará sus enunciados y es allí precisamente donde radica la verdad o falsedad de sus asertos.

Una vez aclarado qué método es el más adecuado para las Ciencias de la Información y, en especial, para las Relaciones Públicas, pasamos a explicitar cuáles son los principios fundamentales de que habría que partir y que son los siguientes:

1. Las Relaciones Públicas son una ciencia cultural, valorativa y finalista.

2. En cuanto ciencia cultural, valorativa y finalista pretenden la realización de un valor: la solidaridad.

3. Esta pretensión se realiza generalmente a través de procesos de comunicación grupales e intergrupales generadores de una reputación social óptima.

\footnotetext{
${ }^{15}$ Ferrater Mora, José, “Diccionario de Filosofía”, voz "forma”, Ediciones Ariel, Barcelona, 2004.
} 
4. Una reputación social óptima es aquella que traduce en cada supuesto concreto los valores de la "norma de cultura". ${ }^{16}$

Para un desarrollo adecuado de esta propuesta metodológica, es necesario ir desenvolviendo los conceptos que involucra, es decir, determinar con rigor y precisión su alcance y naturaleza, basando cada paso en ese "Ars demostrandi" al que aludiera Descartes en su "Discurso del Método".

\section{BIBLIOGRAFÍA}

COSTA, Joan (1993): "Imagen pública, una ingeniería social”, Fundesco, Madrid.

COSTA, Joan (1995): "Comunicación corporativa y revolución de los servicios", Madrid, Ciencias Sociales.

COSTA, Joan (2001): "Imagen Corporativa en el siglo XXI", La Crujía Ediciones, Buenos Aires.

ERBSCHLOE, Michel (2002): "Socially Responsible IT Management".

ETTINGER, K. E. (1961): "Investigación y Relaciones Públicas", México.

FERNÁNDEZ, O. A. B. (1973): "Manual de Relaciones Públicas y Humanas”, Buenos Aires.

FINN, D. (1964): La Empresa y las Relaciones Públicas”, Barcelona.

FLORES BAO, F. (1981): "Relaciones Públicas. Ciencia de la integración humana", Lima.

FLORES BAO, F. (1965): "Fundamentos de Relaciones Públicas", Lima.

FLORES BAO, F. (1969): Teoría y práctica de las Relaciones Públicas", Lima.

HIZZUTO, F. A. (1964): "Relaciones Humanas y Relaciones Públicas” Buenos Aires.

HULA, Kevin W. (2002): “Cabildeo Lobbying”. México.

ILLESCAS, W. D. (1971): “Cómo se practican las Relaciones Públicas”, Buenos Aires. ILLESCAS, W. D. (1975): “Cómo planear las Relaciones Públicas”, Buenos Aires.

JACKSON, Ira y Nelson, Jane (2004): "Profits with Principles".

MONAGHAN, Philip (2003): "Does Reporting Work? The effect of Regulation".

MONTOLÍ LUQUE, L. (1971): “Cómo tener buenas Relaciones Públicas”, Madrid.

NIELANDER, W. A. y Miller, R. W. (1958): “Relaciones Públicas”, Barcelona.

NOGUERO I GRAU, A. (1982): "Relaciones Públicas e Industria de la Persuasión", Barcelona.

PÉREZ SENAC, R. (1975): "Las Relaciones Públicas y el Agro”, Montevideo.

PÉREZ SENAC, R. (1980): "Las Relaciones Públicas en la década de los 80",

MONTEVIDEO RAY, V. (1971): "Cómo iniciarse en Relaciones Públicas", Buenos Aires.

\footnotetext{
${ }^{16}$ Observará el lector que nos referimos a las Relaciones Públicas; ello obedece a que la responsabilidad social corporativa agota el contenido de esta materia, ya que, en última instancia, todas las actividades publirrelacionísticas se enderezan inevitablemente a su satisfacción; en otro supuesto, las Relaciones Públicas se inscriben en un ámbito de frivolidad que repugna a toda persona con un mínimo de inquietud intelectual.
} 
RAYMOND, S. (1970): "Perspectivas de las Relaciones Públicas”, México.

RÍOS SZALAY, J. (1972): "Relaciones Públicas. Su administración en las organizaciones", México.

RÍOS SZALAY, J. (1978): “Relaciones Públicas”, México.

ROBINSON, E. J. (1971): “Comunicación y Relaciones Públicas”, México.

RODARTE, F. F. (1966): “La Empresa y sus Relaciones Públicas”, México.

ROGGERO, G. A. (1970): "Relaciones Públicas", Bilbao.

SARUBA, J. (1972): "Las Relaciones Públicas piden la palabra”, Buenos Aires.

SEIL, M. D. (1982): "Relaciones Públicas y Publicidad", Quito.

SLEE SMITH, P. (1970): "Relaciones Públicas de la Empresa", Bilbao.

SOLANO FLETA, L. (1988): "Tratado de Relaciones Públicas", Madrid.

SOLANO FLETA, L. (1995): "Fundamentos de las Relaciones Públicas", Madrid.

SOLANO FLETA, L. y URZAIZ, J. (1976): "Relaciones Públicas", Madrid.

SPENGLER, Oswaldo (1923): "Der Untergang des Abendlandes". "La Decadencia de Occidente" (trad., 2005).

UGEUX, W. (1976): "Las Relaciones Públicas. Una nueva función social”, Barcelona.

URZAIZ, J. (1971): Teoría y Técnica de las Relaciones Públicas”, Madrid.

URZAIZ, J. (1977): “Ordenación de las Relaciones Públicas”, Madrid.

VARELA, F. J. (1977): "Teoría y práctica de las Relaciones Públicas", Buenos Aires.

XIFRA HERRERAS, J. (1968): “Sociología de las Relaciones Públicas”, Barcelona. 